\title{
Polytropic Inspired Inflation on the Brane
}

\author{
M. Setare, ${ }^{1}$ A. Ravanpak, ${ }^{2}$ and H. Farajollahi ${ }^{3,4}$ \\ ${ }^{1}$ Department of Science of Bijar, University of Kurdistan, Bijar, Iran* \\ ${ }^{2}$ Department of Physics, Vali-e-Asr University, Rafsanjan, Iran \\ ${ }^{3}$ Department of Physics, University of Guilan, Rasht, Iran \\ ${ }^{4}$ School of Physics, University of New South Wales, Sydney, NSW, 2052, Australid
}

(Dated: April 12, 2018)

\begin{abstract}
In this paper, the brane inflationary model inspired by polytropic inflationary idea is studied. In slow-roll approximation and high energy limit, for a chaotic potential, the model is developed and its characteristics is discussed. We obtain explicit expressions for the scalar power spectrum, the tensor-scalar ratio, the scalar spectral index and its running in terms of the polytropic parameters. We find a new constraint on the energy scale of the inflation and the brane tension using the WMAP9 data.
\end{abstract}

Keywords: brane; inflation; slow-roll; polytropic; perturbation

*Electronic address: rezakord@ipm.ir

${ }^{\dagger}$ Electronic address: a.ravanpak@vru.ac.ir

${ }^{\ddagger}$ Electronic address: hosseinf@guilan.ac.ir 


\section{INTRODUCTION}

As is well known, many long-standing problems of the standard hot big bang scenario, such as flatness and horizon problems, can be removed if one consider a very rapidly accelerated expansion phase at the very early stages of the history of the universe which is called inflation [1, 2].

The past few years witnessed a great deal of attention in higher dimensional gravity. It was shown that the fundamental higher-dimensional gravitational scale can be reduced down to the $\mathrm{TeV}$-scale in the presence of a large extra dimension [3] [ 5 . In these theories the standard model of particles is confined to a lower-dimensional brane and only gravity can propagate into the bulk. The effect of the extra dimension induces additional terms in the Friedmann equation on the brane [6 8]. Specially, the existence of a quadratic term in the energy density generally makes it easier to obtain inflation in the early universe [9 13$]$.

On the other hand, the polytropic gas has recently been proposed as an alternative dark energy model for describing the late time acceleration of the universe [14], after that its equation of state is frequently used in various astrophysical situations, such as degenerate white dwarfs, neutron stars, main sequence stars [15], and also in the case of Lane-Emden models [16, 17]. Then, a question was raised; can we have a polytropic inspired inflation like a Chaplygin inspired inflation? Bertolami et al first proposed a phenomenological modification of gravity inspired by the Chaplygin gas equation of state which was then known as Chaplygin inspired inflation [18]. In this scenario the standard inflaton field can be extrapolate to obtain a successful inflationary era with a Chaplygin gas model. After that the authors in [19], extended this procedure to a brane-world Chaplygin inspired inflationary model. Similar works have been done in the context of a Chaplygin inspired inflation using a tachyon scalar field [20, 21]. In a recent work a similar approach was used to introduce a polytropic inspired inflationary model [22]. This scenario was extended to the brane cosmology in which a tachyon field was considered as the inflaton [23]. On the other hand in [24], a generalized polytropic gas equation of state has been introduced and its cosmological applications has been investigated. In the present paper, assuming a canonical scalar field as the inflaton field we study the polytropic inflationary scenario in brane-world models. It can provide the conditions to compare with the results in [22] and [23]. The outline of the paper is as follows. The next section presents a short review of the modified Friedmann 
equation in brane-world model in the presence of a polytropic gas. In Sec III we study the scalar and tensor perturbations in our model and derive the cosmological parameters. In Sec IV we use a chaotic potential in the high-energy limit to obtain explicit expressions for model parameters. Finally, in Sec $[\mathrm{V}$ we present a conclusion.

\section{THE MODEL}

In this section, a five-dimensional brane cosmology is studied with the modified Friedmann equation given by

$$
H^{2}=\kappa \rho_{\phi}\left[1+\frac{\rho_{\phi}}{2 \lambda}\right]+\frac{\Lambda_{4}}{3}+\frac{\xi}{a^{4}}
$$

where $H=\dot{a} / a$ is the Hubble parameter. The $\rho_{\phi}$ and $\Lambda_{4}$ are respectively, the matter field confined to the brane and the four-dimensional cosmological constant. We also assume that $\kappa=8 \pi G / 3=8 \pi /\left(3 m_{p}^{2}\right)$. The influence of the bulk gravitons on the brane is shown in the last term of the equation, where $\xi$ is an integration constant. The four and five-dimensional Planck masses are related through brane tension $\lambda$ in $m_{p}=\sqrt{3 M_{5}^{6} /(4 \pi \lambda)}$, where constrained by nucleosynthesis. The brane tension satisfies the inequality $\lambda>(1 M e V)^{4}$. We suppose that the four-dimensional cosmological constant is zero. In addition, at the beginning of inflation, the last term in (1) vanishes. Thus, the equation (1) reduces to

$$
H^{2}=\kappa \rho_{\phi}\left[1+\frac{\rho_{\phi}}{2 \lambda}\right]
$$

On the other hand, the polytropic fluid which has been proposed as an alternative model for describing the accelerating of the universe [14], has an equation of state of the form

$$
p=K \rho^{1+\frac{1}{n}}
$$

where $K$ and $n$ are constant values called in the literature polytropic constant and polytropic index, respectively. The polytropic constant $K$ can take the positive value for radiation and stiff fluid, the zero value for dust and the negative value for inflationary scenario [14]. The case $n=-\frac{1}{2}$, is motivated by string theory [25 29]. Now, using (3) and conservation equation

$$
\dot{\rho}+3 H(\rho+p)=0
$$

we can obtain

$$
\rho=\left[-K+B a^{\frac{3}{n}}\right]^{-n},
$$


where $B$ is an integration constant. In (41) dot means derivative with respect to the cosmological time.

In this article we will not consider the above equation as a consequence of the polytropic equation of state (3), but we start by studying the modified gravity, where the gravitational dynamic is given by modified Friedmann equation as [30]

$$
H^{2}=\kappa\left[-K+\rho_{\phi}^{\frac{-1}{n}}\right]^{-n}\left[1+\frac{\left(\left[-K+\rho_{\phi}^{\frac{-1}{n}}\right]^{-n}\right)}{2 \lambda}\right] .
$$

In here, $\rho_{\phi}=\frac{1}{2} \dot{\phi}^{2}+V(\phi)$ and $V(\phi)$ is the scalar potential. This modification of energy density is understood from an extrapolation of equation (5) as

$$
\rho=\left[-K+\rho_{m}^{\frac{-1}{n}}\right]^{-n} \rightarrow\left[-K+\rho_{\phi}^{\frac{-1}{n}}\right]^{-n}
$$

where $\rho_{m}$ is the matter energy density [22, 23, 31].

The Klein-Gordon equation for the scalar field confined on the brane is written as

$$
\ddot{\phi}+3 H \dot{\phi}+V^{\prime}=0
$$

and the pressure of a scalar field is expressed as

$$
p_{\phi}=\frac{1}{2} \dot{\phi}^{2}-V(\phi)
$$

During the inflationary epoch the energy density associated to the scalar field is of the order of the potential, i.e. $\rho_{\phi} \sim V$. Assuming the set of slow-roll conditions, i.e. $\dot{\phi}^{2}<<V(\phi)$ and $\ddot{\phi}<<V^{\prime}$, the Friedmann equation (6) reduces to

$$
H^{2} \simeq \kappa\left[-K+V^{\frac{-1}{n}}\right]^{-n}\left[1+\frac{\left(\left[-K+V^{\frac{-1}{n}}\right]^{-n}\right)}{2 \lambda}\right] .
$$

Also equation (8) becomes

$$
3 H \dot{\phi} \simeq-V^{\prime}
$$

Introducing the dimensionless slow-roll parameters, we can write

$$
\varepsilon=-\frac{\dot{H}}{H^{2}} \simeq \frac{V^{\prime 2}}{6 \kappa} V^{\frac{-1}{n}-1}\left[-K+V^{\frac{-1}{n}}\right]^{n-1} \frac{\left[1+\frac{\left(\left[-K+V^{\frac{-1}{n}}\right]^{-n}\right)}{\lambda}\right]}{\left[1+\frac{\left(\left[-K+V^{\frac{-1}{n}}\right]^{-n}\right)}{2 \lambda}\right]^{2}},
$$

and $\delta=\frac{-\ddot{\phi}}{H \dot{\phi}}=\frac{\dot{H}}{H^{2}}+\frac{V^{\prime \prime}}{3 H^{2}}$ as

$$
\delta \simeq \frac{-V^{\prime 2}}{6 \kappa} V^{\frac{-1}{n}-1}\left[-K+V^{\frac{-1}{n}}\right]^{n-1} \frac{\left[1+\frac{\left(\left[-K+V^{\frac{-1}{n}}\right]^{-n}\right)}{\lambda}\right]}{\left[1+\frac{\left(\left[-K+V^{\frac{-1}{n}}\right]^{-n}\right)}{2 \lambda}\right]^{2}}+\frac{V^{\prime \prime}}{3 \kappa\left[-K+V^{\frac{-1}{n}}\right]^{-n}\left[1+\frac{\left(\left[-K+V^{\frac{-1}{n}}\right]^{-n}\right)}{2 \lambda}\right]} .
$$


Also, we can define

$$
\eta \equiv \varepsilon+\delta=\frac{V^{\prime \prime}}{3 \kappa\left[-K+V^{\frac{-1}{n}}\right]^{-n}\left[1+\frac{\left(\left[-K+V^{\frac{-1}{n}}\right]-n\right)}{2 \lambda}\right]},
$$

where in the low-energy limit $\left(-K+\rho_{\phi}^{-1 / n}\right)^{-n} \ll \lambda$, they reduce to the standard form in $[22]$.

The condition under which inflation takes place can be summarized with the parameter $\varepsilon$ satisfying the inequality $\varepsilon<1$, which is analogue to the requirement that $\ddot{a}>0$. This condition could be written in terms of the scalar potential and its derivative $V$ and $V^{\prime}$, as

$$
V^{\prime 2} V^{\frac{-1}{n}-1}\left[1+\frac{\left(\left[-K+V^{\frac{-1}{n}}\right]^{-n}\right)}{\lambda}\right]<6 \kappa\left(\left[-K+V^{\frac{-1}{n}}\right]^{1-n}\right)\left[1+\frac{\left(\left[-K+V^{\frac{-1}{n}}\right]^{-n}\right)}{2 \lambda}\right]^{2} .
$$

Inflation ends when the universe heats up at a time when $\varepsilon \simeq 1$, which implies

$$
V_{f}^{\prime 2} V_{f}^{\frac{-1}{n}-1}\left[1+\frac{\left(\left[-K+V_{f}^{\frac{-1}{n}}\right]^{-n}\right)}{\lambda}\right] \simeq 6 \kappa\left(\left[-K+V_{f}^{\frac{-1}{n}}\right]^{1-n}\right)\left[1+\frac{\left(\left[-K+V_{f}^{\frac{-1}{n}}\right]^{-n}\right)}{2 \lambda}\right]^{2},
$$

where the subscript $f$, has been used for the final values. In high energies when $[-K+$ $\left.V^{\frac{-1}{n}}\right]^{-n} \gg \lambda$, equation (16) becomes

$$
V_{f}^{\prime 2} \simeq \frac{3 \kappa}{2 \lambda} V_{f}^{\frac{1}{n}+1}\left[-K+V_{f}^{\frac{-1}{n}}\right]^{1-2 n} .
$$

Also, the number of e-folds at the end of inflation where indicates the count that scale factor parameter grows by a factor $e$, is given by

$N=\int H d t=\int \frac{H}{\dot{\phi}} d \phi=-3 \int_{\phi_{*}}^{\phi_{f}} \frac{H^{2}}{V^{\prime}} d \phi=-3 \kappa \int_{\phi_{*}}^{\phi_{f}} \frac{\left[-K+V^{\frac{-1}{n}}\right]^{-n}}{V^{\prime}}\left[1+\frac{\left(-K+V^{\frac{-1}{n}}\right)^{-n}}{2 \lambda}\right] d \phi$,

in which $*$, stands for the epoch when the cosmological scales exit the horizon. Also, we can rewrite Eq.(18) in the high-energy limit as

$$
N=-\frac{3 \kappa}{2 \lambda} \int_{V_{*}}^{V_{f}} \frac{\left[-K+V^{\frac{-1}{n}}\right]^{-2 n}}{V^{\prime 2}} d V .
$$

\section{PERTURBATIONS}

In this section we will study the scalar and tensor perturbations for our model following the procedure used in [32]- 34]. It was shown in [35] that the curvature perturbation $\mathcal{R}$ on comoving hypersurfaces is related to the field fluctuation $\delta \phi$ via

$$
\mathcal{R}=\frac{H}{\dot{\phi}} \delta \phi=\frac{H}{\dot{\phi}}\left(\frac{H}{2 \pi}\right) \text {. }
$$


Thus, for a scalar field the power spectrum of the curvature perturbations is given in the slow-roll approximation by the expression $\mathcal{P}_{\mathcal{R}}=\left(\frac{H^{2}}{2 \pi \phi}\right)^{2}[9]$. So, in our model

$$
\mathcal{P}_{\mathcal{R}} \simeq \frac{9 \kappa^{3}}{4 \pi^{2} V^{\prime 2}}\left[-K+V^{\frac{-1}{n}}\right]^{-3 n}\left[1+\frac{\left(\left[-K+V^{\frac{-1}{n}}\right]^{-n}\right)}{2 \lambda}\right]^{3}
$$

Since according to standard notation in the literature the power spectrum is the contribution to the variance per unit logarithmic interval in the wave number $k$, to describe the slope of the power-spectrum we define the scalar spectral index $n_{s}$, through $n_{s}-1=\frac{d \ln \mathcal{P}_{\mathcal{R}}}{d \ln k}$, where the interval in wave number is related to the number of e-folds by the relation $d \ln k(\phi)=d N(\phi)$. From Eq.(21), we get, $n_{s}=1-4 \varepsilon+2 \delta=1-6 \varepsilon+2 \eta$, or equivalently

$$
n_{s}=1-\frac{\left(-K+V^{\frac{-1}{n}}\right)^{n}}{3 \kappa}\left[1+\frac{\left(-K+V^{\frac{-1}{n}}\right)^{-n}}{2 \lambda}\right]^{-1}\left(\frac{3 V^{\prime 2} V^{\frac{-1}{n}-1}}{\left(-K+V^{\frac{-1}{n}}\right)} \frac{\left[1+\frac{\left(-K+V^{\frac{-1}{n}}\right)^{-n}}{\lambda}\right]}{\left[1+\frac{\left(-K+V^{\frac{-1}{n}}\right)^{-n}}{2 \lambda}\right]}-2 V^{\prime \prime}\right) .
$$

Planck 2015 results [36], indicate that there is not any statistically significant running of the scalar spectral index $n_{\text {run }}=d n_{s} / d \ln k$, but we can obtain its relation in our model as

$$
n_{\text {run }}=\frac{4\left(-K+V^{\frac{-1}{n}}\right)}{V^{\frac{-1}{n}-1} V^{\prime}} \frac{\left[1+\frac{\left(-K+V^{\frac{-1}{n}}\right)^{-n}}{2 \lambda}\right]}{\left[1+\frac{\left(-K+V^{\frac{-1}{n}}\right)^{-n}}{\lambda}\right]}\left[3 \varepsilon^{\prime}-\eta^{\prime}\right] \varepsilon
$$

On the other hand, the generation of tensor perturbations during inflation would produce gravitational waves [37] and these perturbations in brane cosmology are more involved since gravitons can propagate into the bulk. The amplitude of tensor perturbations in brane scenario has been calculated in literature [38, 39]

$$
\mathcal{P}_{g}=24 \kappa\left(\frac{H}{2 \pi}\right)^{2} F^{2}(x),
$$

where $x=H m_{p} \sqrt{3 /(4 \pi \lambda)}$ and

$$
F(x)=\left[\sqrt{1+x^{2}}-x^{2} \sinh ^{-1}\left(\frac{1}{x}\right)\right]^{-\frac{1}{2}} .
$$

In our model it reduces to

$$
\mathcal{P}_{g} \simeq \frac{6 \kappa^{2}}{\pi^{2}}\left[-K+V^{\frac{-1}{n}}\right]^{-n}\left[1+\frac{\left(\left[-K+V^{\frac{-1}{n}}\right]^{-n}\right)}{2 \lambda}\right] F^{2}(x)
$$

From expressions (21) and (26) we write the tensor-scalar ratio as

$$
r=\left.\left.\left(\frac{\mathcal{P}_{g}}{\mathcal{P}_{\mathcal{R}}}\right)\right|_{k=k_{*}} \simeq \frac{8 V^{\prime 2}}{3 \kappa} \frac{\left(-K+V^{\frac{-1}{n}}\right)^{2 n}}{\left(1+\frac{\left(-K+V^{\frac{-1}{n}}\right)^{-n}}{2 \lambda}\right)^{2}} F^{2}(V)\right|_{k=k_{*}} .
$$

Here, $k_{*}$ is referred to $k=H a$, the value when the universe scale crosses the Hubble horizon during inflation. 


\section{CHAOTIC POTENTIAL IN THE HIGH ENERGY LIMIT}

Let us consider an inflaton scalar field $\phi$, with a chaotic potential as $V=m^{2} \phi^{2} / 2$, where $m$ is the mass of the scalar field. In the following, we develop our model in the high-energy limit, i.e. $\left(-K+V^{\frac{-1}{n}}\right)^{-n} \gg \lambda$.

Since for the potential under consideration, $V^{\prime 2}=2 m^{2} V$, using Mathematica we obtain from Eq.(19)

$$
N=\frac{3 \kappa}{8 m^{2} \lambda}\left[h\left(V_{*}\right)-h\left(V_{f}\right)\right]
$$

in which

$$
h(V)=V_{2}^{2} F_{1}\left(2 n, 2 n ; 2 n+1 ; K V^{\frac{1}{n}}\right),
$$

and ${ }_{2} F_{1}$, is a hypergeometric function. Also, Eq.(21) reduces to

$$
\mathcal{P}_{\mathcal{R}} \approx \frac{9 \kappa^{3}}{64 \pi^{2} m^{2} \lambda^{3} V}\left(-K+V^{\frac{-1}{n}}\right)^{-6 n},
$$

and from Eq.(221) and Eq.(23) we obtain

$$
n_{s} \approx 1-\frac{4 m^{2} \lambda}{3 \kappa}\left(-K+V^{\frac{-1}{n}}\right)^{2 n}\left(\frac{6 V^{\frac{-1}{n}}}{\left(-K+V^{\frac{-1}{n}}\right)}-1\right),
$$

and

$$
n_{\text {run }} \approx-\frac{32 m^{4} \lambda^{2}}{9 n \kappa^{2}}\left(-K+V^{\frac{-1}{n}}\right)^{4 n-2}\left[(n-3) K+5 n V^{\frac{-1}{n}}\right] V^{\frac{-1}{n}},
$$

respectively.

In the case of tensor perturbations, one can check that in the high energy limit, we have $F^{2}(x) \approx \frac{3}{2} x[40,41]$, where in our model $x=\frac{1}{\lambda}\left[-K+V^{\frac{-1}{n}}\right]^{-n}$. Then, the power spectrum of tensor perturbation can be rewritten as

$$
\mathcal{P}_{g}=\frac{9 \kappa^{2}}{2 \pi^{2} \lambda^{2}}\left[-K+V^{\frac{-1}{n}}\right]^{-3 n},
$$

and then the tensor to scalar ratio becomes

$$
r \approx \frac{32 m^{2} \lambda V}{\kappa}\left(-K+V^{\frac{-1}{n}}\right)^{3 n} .
$$

Using Eqs.(30) and (34), one can solve $K$ and $m$, in terms of $\mathcal{P}_{\mathcal{R}}, r, V$ and $\lambda$ as follows

$$
K=V^{\frac{-1}{n}}-\left[\frac{2 \pi^{2} \lambda^{2} \mathcal{P}_{\mathcal{R}} r}{9 \kappa^{2}}\right]^{\frac{-1}{3 n}},
$$

and

$$
m=\frac{\pi r}{12} \sqrt{\frac{\lambda \mathcal{P}_{\mathcal{R}}}{\kappa V}} .
$$


As we mentioned in Sec.II, we know from [14] that for an inflationary universe $K<0$. Thus, from (35), the ratio $\frac{V^{3}}{\lambda^{2}}$, satisfies the inequality below

$$
\frac{V^{3}}{\lambda^{2}}>\frac{2 \pi^{2} r \mathcal{P}_{\mathcal{R}}}{9 \kappa^{2}}
$$

where according to the results of WMAP nine year data [42], if we choose $\mathcal{P}_{\mathcal{R}}=2.464 \times 10^{-9}$ and $r=0.13$, a lower limit for this ratio can be obtained as $V_{*}^{3} / \lambda^{2}>7.02 \times 10^{-10}$, in which we have assumed $\kappa=1$. We should note that in [19] a lower limit, $V_{*}^{3} / \lambda^{2}>7.2 \times 10^{-11} m_{p}^{4}$, and in [21] an upper limit, $V_{*}^{3} / \lambda^{2}<2.4 \times 10^{-11} m_{p}^{4}$, for this ratio has been obtained but using WMAP5 data and in a Chaplygin inspired brane inflationary scenario, using respectively a standard and a tachyon scalar field. For comparison, we can rewrite our result in terms of $m_{p}^{4}$, as $V_{*}^{3} / \lambda^{2}>1.001 \times 10^{-11} m_{p}^{4}$.

\section{SUMMARY}

In this paper we studied polytropic inflationary model in the context of a braneworld scenario. We obtained the dimensionless slow-roll parameters. The condition under which inflation takes place is discussed by using the parameter $\varepsilon$ satisfying the inequality $\varepsilon<$ 1. This condition is expressed in terms of the scalar potential and its derivative as given by Eq.(15). Then, we studied the scalar and tensor perturbations and derived the power spectrum of the curvature perturbations in the slow-roll approximation and an expression for the corresponding scalar spectrum index $n_{s}$ and its running $n_{\text {run }}$. Then, we considered an inflaton scalar field with a chaotic potential and developed the model in the high-energy limit. We obtained the scalar power spectrum, the tensor-scalar ratio, the scalar spectral index and its running in terms of polytropic constant $K$, polytropic index $n$, mass of the inflaton field $m$, brane tension $\lambda$ and the potential $V$. By using these relations and observational data from WMAP9 we obtained a new lower limit for the ratio $V^{3} / \lambda^{2}$.

[1] A. Guth, Phys. Rev. D 23, 347 (1981).

[2] A. Albrecht and P. J. Steinhardt, Phys. Rev. Lett. 48, 1220 (1982).

[3] N. Arkani-Hamed, S. Dimopoulos, and G. Dvali, Phys. Lett. B 429, 263 (1998).

[4] N. Arkani-Hamed, S. Dimopoulos, and G. Dvali, Phys. Rev. D 59, 086004 (1999). 
[5] I. Antoniadis, N. Arkani-Hamed, S. Dimopoulos, and G. Dvali, Phys. Lett. B 436, 257 (1998).

[6] P. Binetruy, C. Deffayet and D. Langlois, Nucl. Phys. B 565, 269 (2000).

[7] P. Binetruy, C. Deffayet, U. Ellwanger and D. Langlois, Phys. Lett. B 477, 285 (2000).

[8] T. Shiromizu, K. Maeda and M. Sasaki, Phys. Rev. D 62, 024012 (2000).

[9] R. Maartens, D. Wands, B. A. Bassett and I. P. C. Heard, Phys. Rev. D 62, 041301 (2000).

[10] J. M. Cline, C. Grojean and G. Servant, Phys. Rev. Lett. 83, 4245 (1999).

[11] C. Csaki, M. Graesser, C. Kolda and J. Terning, Phys. Lett. B 462, 34 (1999).

[12] D. Ida, JHEP. 0009, 014 (2000).

[13] R. N. Mohapatra, A. Perez-Lorenzana and C. A. de S. Pires, Phys. Rev. D 62, 105030 (2000).

[14] U. Mukhopadhyay and S. Ray, Mod. Phys. Lett. A 23, 3198, (2008).

[15] J. Christensen-Dalsgard, Lecture Notes on Stellar Structure and Evolution, 6th edn. (Aarhus Univ. Press, Aarhus, 2004).

[16] R. N. Tiwari, J. R. Rao and R. R. Kanakamedala, Phys. Rev. D 34, 1205 (1986).

[17] S. Ray, Astrophys. Space Sci. 280, 345 (2002).

[18] O. Bertolami and V. Duvvuri, Phys. Lett. B 640, 121 (2006).

[19] R. Herrera, Phys. Lett. B 664, 149 (2008).

[20] S. del Campo and R. Herrera, Phys. Lett. B 660, 282-286 (2008).

[21] R. Herrera, Gen. Rel. Grav. 41, 1259-1271 (2009).

[22] M. R. Setare and F. Darabi, Chin. J. Phys. 51, 3, 427 (2013).

[23] M. R. Setare and V. Kamali, Cent. Eur. J. Phys. 11, 5, 545 (2013).

[24] P. H. Chavanis, Eur. Phys. J. Plus 129, 222 (2014).

[25] L. Randall and R. Sundrum, Phys. Rev. Lett. 83, 4690 (1999).

[26] D. Dvali, G. Gabadadze and M. Porrati, Phys. Lett. B 485, 208 (2000).

[27] K. Freese and M. Lewis, Phys. Lett. B 540, 1 (2002).

[28] R. Maartens, Lect. Notes Phys. 653, 213 (2004).

[29] A. Lue, Phys. Rep. 423, 1 (2006).

[30] R. Maartens, Reference Frames and Gravitomagnetism, 93, (2001); gr-qc/0101059.

[31] M. R. Setare, M. J. S. Houndjo and V. Kamali, Int. J. Mod. Phys. D 22, 8, 1350041 (2013).

[32] S. del Campo, R. Herrera and A. Toloza, Phys. Rev. D 79, 083507 (2009).

[33] S. del Campo and R. Herrera, Phys. Lett. B 670, 266-270 (2009).

[34] H. Farajollahi and A. Ravanpak, Phys. Rev. D 84, 084017 (2011). 
[35] D. Wands, K. A. Malik, D. H. Lyth and A. R. Liddle, Phys. Rev. D 62, 043527 (2000).

[36] P. A. R. Ade et al. [Planck Collaboration], A\&A, 594, A20 (2016).

[37] B. A. Bassett, S. Tsujikawa and D. Wands, Rev. Mod. Phys. 78, 537 (2006).

[38] D. Langlois, R. Maartens and D. Wands, Phys. Lett. B 489, 259 (2000).

[39] M. Sami, N. Savchenko and A. Toporensky, Phys. Rev. D 70, 123528 (2004)

[40] J. E. Lidsey, Lect. Notes Phys. 646, 357 (2004).

[41] M. Naciri, et al., Adv. Studies Theor. Phys. 8, 277 (2014).

[42] C. L. Bennett, et al., Astrophys. J. Suppl. 208, 20B (2013). 